\title{
Covid-19 - Experience in Costa Rica
}

\author{
Alcibey Alvarado* \\ Internal Medicine and Neumology Clínica de Diagnóstico Médico. San José, Costa Rica
}

\section{To The Editor}

Costa Rica (CR) is a mountainous country with a tropical climate, located in Central America, limiting Nicaragua to the North and Panama to the South, with a surface area of $50,900 \mathrm{~km}^{2}$ and 5 million inhabitants, with coasts in both oceans. According to the Multidimensional Poverty Index, the percentage of households living in poverty is $20 \%$. However, CR invests $7.3 \%$ of its Gross Domestic Product in health, exceding WHO standards.

In CR, 951 cases of SARS-CoV-2 (rRT-CPR) have been confirmed $(05 / 26 / 2020)$, with 10 deaths, and 628 patients recovered $(63 \%)$ and 313 active cases. There are 15 hospitalized patients and only 2 of them in Intensive Care (227 ICU beds available). The case fatality rate is $1.05 \%$ and the crude mortality rate is 2 per million inhabitants. As a small, third-world, low-income country, these have been gratifying results. We believe that the actions and tools CR uses to confront this pandemic could serve as an example for other countries.

The first successful decision was made by the government. The President of the Republic responsibly ceded the field to science. The first case was diagnosed on 03/06/2020 and 48 hours later the CEO (emergency operations center) was created, which included a series of institutions that provide public services. Ten days after the first case was confirmed and with 41 registered patients, the government declared a state of emergency for Covid-19. The border closure was implemented on $03 / 16 / 2020$, exhibiting a very high reaction speed. From an operational point of view, two state institutions manage health logistics: the Costa Rican Social Security Fund (CCSS) and the Ministry of Health. The first represented by its executive president and the medical manager and the second by the Minister of Health. The CCSS is the state entity responsible for managing health in CR. It was founded in 1941 and consists of a staggered and articulated system of echelons for health care. This entity offers health coverage to more than $95 \%$ of the country's population (universal health system). This contrasts with the rest of the Latin American region in which $30 \%$ of the population does not have access to health services. It currently has 23 national, specialized, regional and peripheral hospitals. Basic primary care is administered in clinics known as EBAIS (basic teams for comprehensive health care) of which there are approximately 1,100 distributed throughout the geography. Every EBAIS has a doctor, nurse, pharmacy service and other technical services. $76 \%$ of the CCSS consultations are evacuated in these clinics, which reflect the importance of the first level of care in the health services network. It is in these EBAIS where the majority of the suspected patients of Covid-19 consult for the first time, close and daily follow-up is given in the communities to the patients, suspicious cases and contact tracing is carried out. If the patient qualifies as a suspect, they are sent to the corresponding Health Area (106 throughout the country with a more complex design). In an isolated room from usual care (to keep the patients separated), the nasopharyngeal swab is taken and sent to the corresponding hospital or to INCIENSA (Costa Rican Institute of Research and Teaching in Nutrition and Health) to process the sample by rRT-PCR (the test and the treatment are free). Incidentally, the genomic department of INCIENSA is the only laboratory that has sequenced the SARSCov-2 virus genome in the Latin American area. There is also a home sampling service. To expand the sampling coverage, some EBAIS have been trained to obtain swabs and some private laboratories have been authorized, but supervised by the Ministry of Health. The "sentinel surveillance" system is being used to search for infected people and that, for some reason, does not meet the definition of a suspected case, adding health centers to the application of diagnostic tests. This system has reduced the saturation of hospital emergency services with patients with symptoms suggestive of Covid-19 disease. CENARE (a National Rehabilitation Center) was quickly redesigned and in less than 15 days it was enabled to admit patients only and exclusively with Covid-19. The center has 88 beds, the appropriate equipment and health personnel, to attend patients of low to medium complexity. The basic idea is to have patients with Covid-19 centralized and avoid occupying beds in various hospitals in the country. Cases are detected early when symptoms start to appear, this being one of the possible reasons why there are fewer serious cases. Only $9.5 \%$ of cases are redirected to hospitals and only $1.9 \%$ requires admission to ICU. The Costa Rican health model, based on two institutions with a centralized command has been basic and efficient in managing this event.

A second crucial aspect is the availability of water. $98 \%$ of the population has access to drinking water, essential for hand washing. In the rest of the Central American region, only $27 \%$ of rural areas and $72 \%$ of urban areas have access to drinking water (United Nations data).

A third element is population. Primary education was declared free and compulsory in Costa Rica in 1869 and the army was abolished in 1948. These 2 socio-political elements helped to form a solid democracy as the basis of current Costa Rican society. The population's health education is part of that legacy. When the government announced the containment and mitigation measures, except for some violations, the population began to accept them. Frequent handwashing, coughing and sneezing protocol, trying to touch your face less, house confinement, telecommuting, class suspension, mass meetings suspension, tourist activities, and vehicle restriction paid off. Attendance at shops, public spaces, beaches and resorts was reduced by $84 \%$. The population interpreted and understood that in the absence of an effective and proven antiviral treatment and a vaccine, the behavior of the population was vital for the control of the pandemic. In this sense, the

*Correspondence to: Alcibey Alvarado. Clínica de Diagnóstico Médico. Torre Médica. 3 piso. Paseo Colón, San José, Costa Rica, Tel: 50622237134/5062256 6439/50687351858; Fax: 50622216754; E-mail: alcialvagonza@yahoo.com.mx

Received: May 26, 2020; Accepted: June 15, 2020; Published: June 18, 2020 
government's decision to inform the population has been laudable. The daily report, on television (from the presidential house or the Ministry of Health), of the evolution of the problem and of the actions that have been implemented has increased the credibility of the population in the guidelines and therefore of adherence to them. There is no community transmission as most of the epidemiological nexus have been identified in confirmed cases (above 95\%).

Without high immunity rates in the population, CR remains fragile to the virus. The possibility of aggressive sampling for the detection of new cases, particularly of asymptomatic patients who have not yet been detected, is being implemented. rRT-CPR is applied to absolutely all carriers that enter with supplies or merchandise through both land borders. The use of cell phones for case tracking and contact identification was implemented. The idea is to use an app called EDUS (unique digital health record) of which there are already 2.1 million downloads that capture the identification of cases, storing the information in a private cloud, under a contract with Microsoft and managed by technical health teams, subject to professional secrecy. We are working on the local production of ventilators (University of Costa Rica and Costa Rica Technological Institute [TEC]) due to the hypothetical possibility of an "spillover" of the fleet of available mechanical ventilators and on the production of antibodies in approximately 4 months for the most severe cases, using plasma of recovered patients and antibodies obtained from horse plasma by inoculation of viral proteins to them (Clodomiro Picado Institute).
An additional problem that CR has is the border with Nicaragua. This border of more than $309 \mathrm{~km}$ is porous for the passage of legal and illegal immigrants. Border health control is critical as the neighboring country has no concerted government action to deal with the pandemic, and border surveillance has been strengthened. Nicaraguan official report is not reliable. Independent Nicaraguan sources assure that there is already community transmission. It is obvious that what happens and does, or does not do, neighboring countries, may end up affecting Costa Rican health policy.

Identifying new cases, tracing contacts, and limiting transmission is critical to lifting the curtailment of some social measures, before effective treatment and vaccination are available.

\section{Authors contribution}

This work was only carried out by the author. Author AA contributed in the planning, data collection, data analysis, writing and critical review. AA read and approved the final manuscript.

\section{Source of economic support}

No.

\section{Conflict of interest}

No.

Copyright: (C2020 Alvarado A. This is an open-access article distributed under the terms of the Creative Commons Attribution License, which permits unrestricted use, distribution, and reproduction in any medium, provided the original author and source are credited. 